\title{
On the Content Security Policy Violations due to the Same-Origin Policy
}

\author{
Dolière Francis Some \\ Université Côte d'Azur \\ Inria, France \\ doliere.some@inria.fr
}

\author{
Nataliia Bielova \\ Université Côte d'Azur \\ Inria, France \\ nataliia.bielova@inria.fr
}

\author{
Tamara Rezk \\ Université Côte d'Azur \\ Inria, France \\ tamara.rezk@inria.fr
}

\begin{abstract}
Modern browsers implement different security policies such as the Content Security Policy (CSP), a mechanism designed to mitigate popular web vulnerabilities, and the Same Origin Policy (SOP), a mechanism that governs interactions between resources of web pages.

In this work, we describe how CSP may be violated due to the SOP when a page contains an embedded iframe from the same origin. We analyse 1 million pages from 10,000 top Alexa sites and report that at least $31.1 \%$ of current CSPenabled pages are potentially vulnerable to CSP violations. Further considering real-world situations where those pages are involved in same-origin nested browsing contexts, we found that in at least $23.5 \%$ of the cases, CSP violations are possible.

During our study, we also identified a divergence among browsers implementations in the enforcement of CSP in srcdoc sandboxed iframes, which actually reveals a problem in Gecko-based browsers CSP implementation. To ameliorate the problematic conflicts of the security mechanisms, we discuss measures to avoid CSP violations.
\end{abstract}

\section{INTRODUCTION}

Modern browsers implement different specifications to securely fetch and integrate content. One widely used specification to protect content is the Same Origin Policy (SOP) 3]. SOP allows developers to isolate untrusted content from a different origin. An origin here is defined as scheme, host, and port number. If an iframe's content is loaded from a different origin, SOP controls the access to the embedder resources. In particular, no script inside the iframe can access content of the embedder page. However, if the iframe's content is loaded from the same origin as the embedder page, there are no privilege restrictions w.r.t. the embedder resources. In such a case, a script executing inside the iframe can access content of the embedder webpage. Scripts are considered trusted and the iframe becomes transparent from a developer view point. A more recent specification to

(C) 2017 International World Wide Web Conference Committee (IW3C2), published under Creative Commons CC BY 4.0 License. WWW 2017, April 3-7, 2017, Perth, Australia. ACM 978-1-4503-4913-0/17/04. http://dx.doi.org/10.1145/3038912.3052634

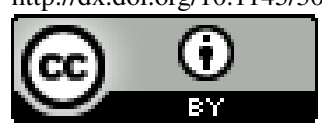

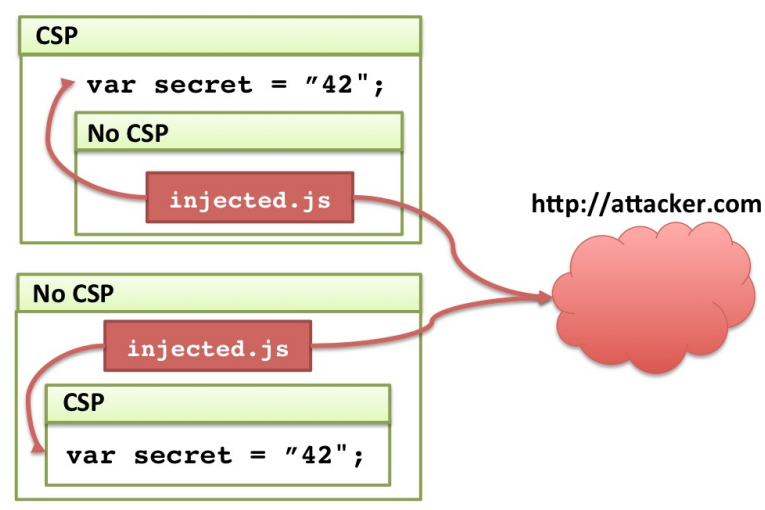

Figure 1: An XSS attack despite CSP.

protect content in web pages is the Content Security Policy (CSP) 19. The primary goal of CSP is to mitigate cross site scripting attacks (XSS), data leaks attacks, and other types of attacks. CSP allows developers to specify, among other features, trusted domain sources from which to fetch content. One of the most important features of CSP, is to allow a web application developer to specify trusted JavaScript sources. This kind of restriction is meant to permit execution of only trusted code and thus prevent untrusted code to access content of the page.

In this work, we report on a fundamental problem of CSP. CSP 28, defines how to protect content in an isolated page. However, it does not take into consideration the page's context, that is its embedder or embedded iframes. In particular, CSP is unable to protect content of its corresponding page if the page embeds (using the src attribute) an iframe of the same origin. The CSP policy of a page will not be applied to an embedded iframe. However, due to SOP, the iframe has complete access to the content of its embedder. Because same origin iframes are transparent due to SOP, this opens loopholes to attackers whenever the CSP policy of an iframe and that of its embedder page are not compatible (see Fig. 1).

We analysed 1 million pages from the top 10,000 Alexa sites and found that $5.29 \%$ of sites contain some pages with CSPs (as opposed to $2 \%$ of home pages in previous studies [5]). We have identified that in $94 \%$ of cases, CSP may be violated in presence of the document.domain API and in $23.5 \%$ of cases CSP may be violated without any assumptions (see Table 3 ).

During our study, we also identified a divergence among browsers implementations in the enforcement of CSP 28, in 
sandboxed iframes embedded with srcdoc. This actually reveals an inconsistency between the CSP and HTML5 sandbox attribute specification for iframes.

We identify and discuss possible solutions from the developer point of view as well as new security specifications that can help prevent this kind of CSP violations. We have made publicly available the dataset that we used for our results in 2. We have installed an automatic crawler to recover the same dataset every month to repeat the experiment taking into account the time variable. An accompanying technical report with a complete account of our analyses can be found at 18 .

In summary, our contributions are: (i) We describe a new class of vulnerabilities that lead to CSP violations. (Section 11. (ii) We perform a large and depth scale crawl of top sites, highlighting CSP adoption at sites-level, as well as sites origins levels. Using this dataset, we report on the possibilities of CSP violations between the SOP and CSP in the wild. (Section 3). (iii) We propose guidelines in the design and deployment of CSP. (Section 3.4). (iv) We reveal an inconsistency between the CSP specification and HTML5 sandbox attribute specification for iframes. Different browsers choose to follow different specifications, and we explain how any of these choices can lead to new vulnerabilities. (Section 5).

\section{CONTENT SECURITY POLICY AND SOP}

The Content Security Policy (CSP) 19 is a mechanism that allows programmers to control which client-side resources can be loaded and executed by the browser. CSP (version 2) is an official W3C candidate recommendation 28, and is currently supported by major web browsers. CSP is delivered in the Content-Security-Policy HTTP response header, or in a <meta> element of HTML.

CSP applicability A CSP delivered with a page controls the resources of the page. However it does not apply to the page's embedding resources 28. As such, CSP does not control the content of an iframe even if the iframe is from the same origin as the main page according to SOP. Instead, the content of the iframe is controlled by the CSP delivered with it, that can be different from the CSP of the main page.

CSP directives CSP allows a programmer to specify which resources are allowed to be loaded and executed in the page. These resources are defined as a set of origins and known as a source list. Additionally to controlling resources, CSP allows to specify allowed destinations of the AJAX requests by the connect-src directive. A special header Content-Security-Policy-Report-Only configures a CSP in a report-only mode: violations are recorded, but not enforced. The directive default-src is a special fallback directive that is used when some directive is not defined. The directive frame-ancestors (meant to supplant the HTTP X-Frame-Options header 28]), controls in which pages the current page may be included as an iframe, to prevent clickjacking attacks 16. See Table 1 for the most commonly used CSP directives 22].

Source lists CSP source list is traditionally defined as a whitelist indicating which domains are trusted to load the content, or to communicate. For example, a CSP from Listing 1 allows to include scripts only from third.com, requires to load frames only over HTTPS, while other resource types can only be loaded from the same hosting domain.

1 Content-Security-Policy: default-src 'self ;

\begin{tabular}{l|l} 
Directive & Controlled content \\
\hline script-src & Scripts \\
default-src & All resources (fallback) \\
style-src & Stylesheets \\
img-src & Images \\
font-src & Fonts \\
connect-src & XMLHttpRequest, WebSocket or \\
& EventSource \\
object-src & Plug-in formats (object, embed) \\
report-uri & URL where to report CSP violations \\
media-src & Media (audio, video) \\
child-src & Documents (frames), [Shared] Workers \\
frame-ancestors & Embedding context
\end{tabular}

Table 1: Most common CSP directives 22.

2 script-src third.com; child-src https:

Listing 1: Example of a CSP policy.

A whitelist can be composed of concrete hostnames (third.com), may include a wildcard $*$ to extend the policy to subdomains (*.third.com), a special keyword 'self' for the same hosting domain, or 'none' to prohibit any resource loading.

Restrictions on scripts Directive script-src is the most used feature of CSP in today's web applications 22. It allows a programmer to control the origin of scripts in his application using source lists. When the script-src directive is present in CSP, it blocks the execution of any inline script, JavaScript event handlers and APIs that execute string data code, such as eval() and other related APIs. To relax the CSP, by allowing the execution of inline <script> and JavaScript event handlers, a script-src whitelist should contain a keyword 'unsafe-inline'. To allow eval()-like APIs, the CSP should contain a 'unsafeeval' keyword. Because 'unsafe-inline' allows execution of any inlined script, it effectively removes any protection against XSS. Therefore, nonces and hashes were introduced in CSP version 2 28, allowing to control which inline scripts can be loaded and executed.

Sandboxing iframes Directive sandbox allows to load resources but execute them in a separate environment. It applies to all the iframes and other content present on the page. An empty sandbox value creates completely isolated iframes. One can selectively enable specific features via allow-* flags in the directive's value. For example, allow-scripts will allow executions of scripts in an iframe, and allow-sameorigin will allow iframes to be treated as being from their normal origins.

\section{Same-Site and Same-Origin Definitions.}

In our terminology, we distinguish the web pages that belong to the same site from the pages that belong to the same origin. By page we refer to any HTML document - for example, the content of an iframe we call iframe page. In this case, the page that embeds an iframe is called a parent page or embedder.

By site we refer to the highest level domain that we extract from Alexa top 10,000 sites, usually containing the domain name and a TLD, for example main.com. All the pages that belong to a site, and to any of its subdomains as sub.main.com, are considered same-site pages.

According to the Same Origin Policy, an origin of a page is scheme, host and port of its URL. For example, in http: 
//main.com:81/dir/p.html, the scheme is "http", the host is "main.com" and the port is 81 .

\subsection{CSP violations due to SOP}

Consider a web application, where the main page A.html and its iframe B.html are located at http://main.com, and therefore belong to the same origin according to the sameorigin policy. A.html, shown in Listing 2, contains a script and an iframe from main.com. The local script secret.js contains sensitive information given in Listing 3 . To protect against XSS, the developer behind http://main.com have installed the CSP for its main page A.html, shown in Listing 4.

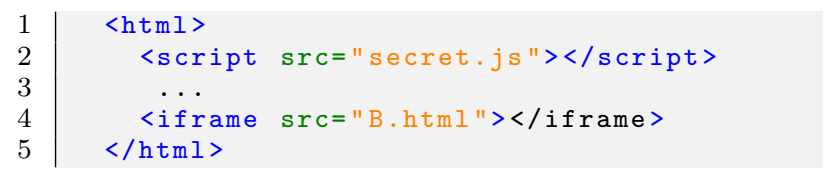

Listing 2: Source code of http://main.com/A.html.

1 var secret $=" 42 "$;

Listing 3: Source code of secret.js.

\begin{tabular}{l|l}
1 & Content-Security-Policy: default-src 'none \\
2 & script-src 'self'; child-src 'self'
\end{tabular}

Listing 4: CSP of http://main.com/A.html.

This CSP provides an effective protection against XSS:

\subsubsection{Only parent page has CSP}

According to the latest version of $\mathrm{CSP}^{1}$ only the CSP of the iframe applies to its content, and it ignores completely the CSP of the including page. In our case, if there is no CSP in B.html then its resource loading is not restricted. As a result, an iframe B.html without CSP is potentially vulnerable to XSS, since any injected code may be executed within B.html with no restrictions. Assume B.html was exploited by an attacker injecting a script injected.js. Besides taking control over B.html, this attack now propagates to the including page A.html, as we show in Fig. 1] The XSS attack extends to the including parent page because of the inconsistency between the CSP and SOP. When a parent page and an iframe are from the same origin according to SOP, a parent and an iframe share the same privileges and can access each other's code and resources.

For our example, injected.js is shown in Listing 5 .

This script executed in B.html retrieves the secret value from its parent page (parent.secret) and transmits it to an attacker's server http://attacker . com via XMLHttpRequest 2

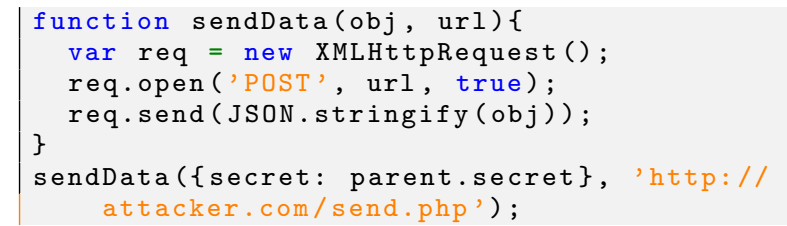

Listing 5: Source code of injected.js.

\footnotetext{
$\sqrt[1]{\text { https : //www.w3.org/TR/CSP2/\#which-policy-applies }}$ ${ }^{2}$ The XMLHttpRequest is not forbidden by the SOP for B.html because an attacker has activated the CrossOrigin Resource Sharing mechanism 21. on her server http://attacker.com.
}

A straightforward solution to this problem is to ensure that the protection mechanism for the parent page also propagates to the iframes from the same domain. Technically, it means that the CSP of the iframe should be the same or more restrictive than the CSP of the parent. In the next example we show that this requirement does not necessarily prevent possible CSP violations due to SOP.

\subsubsection{Only iframe page has CSP}

Consider a different web application, where the including parent page A.html does not have a CSP, while its iframe B.html contains a CSP from Listing 4. In this example, B.html, shown in Listing 6 now contains some sensitive information stored in secret.js (see Listing 3 .

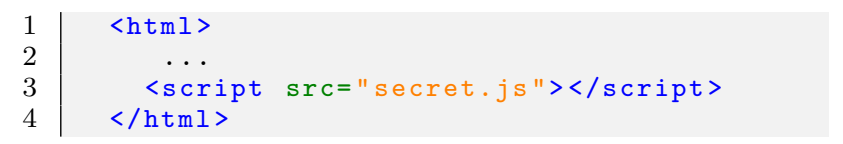

Listing 6: Source code of http://main.com/B.html.

Since the including page A.html now has no CSP, it is potentially vulnerable to XSS, and therefore may have a malicious script injected.js. The iframe B.html has a restrictive CSP, that effectively contributes to protection against XSS. Since A.html and B.html are from the same origin, the malicious injected script can profit from this and steal sensitive information from B.html. For example, the script may call the sendData function with the secret information:

$$
1 \text { sendData ( } \text { secret: children [0]. secret }\} \text {, }
$$
http: // attacker.com/send.php');

Thanks to SOP, the script injected.js fetches the secret from it's child iframe B.html and sends it to http: //attacker. com.

\subsubsection{CSP violations due to origin relaxation}

A page may change its own origin with some limitations. By using the document.domain API, the script can change its current domain to a superdomain. As a result, a shorter domain is used for the subsequent origin check $\$^{3}$

Consider a slightly modified scenario, where the main page A.html from http://main.com includes an iframe B.html from its sub-domain http://sub.main.com. Any script in B.html is able to change the origin to http://main.com by executing the following line:

1 document.domain = "main.com";

If A.com is willing to communicate with this iframe, it should also execute the above-written code so that the communication with B.html will be possible. The content of B.html is now treated by the web browser as the same-origin content with A.html, and therefore any of the previously described attacks become possible.

\subsubsection{Categories of CSP violations due to SOP}

We distinguish three different cases when the CSP violation might occur because of SOP:

Only parent page or only iframe has CSP A parent page and an iframe page are from the same origin, but only one of them contains a CSP. The CSP may be violated due to the unrestricted access of a page without CSP

3 https://developer.mozilla.org/en-US/docs/Web/ Security/Same-origin_policy\#Changing_origin 
to the content of the page with CSP. We demonstrated this example in Sections 2.1.1 and 2.1.2.

Parent and iframe have different CSPs A parent page and an iframe page are from the same origin, but they have different CSPs. Due to SOP, the scripts from one page can interfere with the content of another page thus violating the CSP.

CSP violation due to origin relaxation A parent page and an iframe page have the same higher level domain, port and scheme, but however they are not from the same origin. Either CSP is absent in one of them, or they have different CSPs - in both cases CSP may be violated because the pages can relax their origin to the high level domain by using document.domain API, as we have shown in Section 2.1.3.

\section{EMPIRICAL STUDY OF CSP VIOLATIONS}

We have performed a large-scale study on the top 10,000 Alexa sites to detect whether CSP may be violated due to an inconsistency between CSP and SOP. For collecting the data, we have used CasperJS 15 on top of PhantomJS headless browser 8. The User-Agent HTTP header was instantiated as a recent Google Chrome browser.

\subsection{Methodology}

The overview of our data collection and CSP comparison process is given in Figure 2 The main difference in our data collection process from previous works on CSP measurements in the wild 22,5 is that we crawl not only the main pages of each site, but also other pages. First, we collect pages accessible through links of the main page and pointing to the same site. Second, to detect possible CSP violations due to SOP, we have collected all the iframes present on the home pages and linked pages.

\subsubsection{Data Collection}

We run PhantomJS using as user agent Mozilla/5.0 (X11; Linux x86_64) AppleWebKit/537.36 (KHTML, like Gecko) Chrome 51.0.2704.63 Safari/537.36. The study was performed on an internal cluster of 200 cores, using OpenMP to benefit from parallelization.

Home Page Crawler For each site in top 10,000 Alexa list, we crawl the home page, parse its source code and extract three elements: (1) a CSP of the site's home page stored in HTTP header as well as in <meta> HTML tag; we denote the CSPs of the home page by $\mathcal{C}$; $(2)$ to extract more pages from the same site, we analyse the source of the links via $<a$ href $=\ldots>$ tag and extract URLs that point to the same site, we denote this list by $L$. (3) we collect URLs of iframes present on the home page via $\langle$ iframe $\mathrm{src}=\ldots\rangle$ tag and record only those belonging to the same site, we denote this set by $\mathcal{F}$.

Page Crawler We crawl all the URLs from the list of pages $L$, and for each page we repeat the process of extraction of CSP and relevant iframes, similar to the steps (1) and (3) of the home page crawler. As a result, we get a set of CSPs of linked pages $\mathcal{C}_{L}$ and a set of iframes URLs $\mathcal{F}_{L}$ that we have extracted from the linked pages in $L$.

Iframe Crawler

For every iframe URL present in the list of home page iframes $\mathcal{F}_{H}$, and in the list of linked pages iframes $\mathcal{F}_{L}$, we extract their corresponding CSPs and store in two sets: $\mathcal{C}_{F}$ for home page iframes and $\mathcal{C}_{L F}$ for linked page iframes.

\subsubsection{CSP adoption analysis}

Since CSP is considered an effective countermeasure for a number of web attacks, programmers often use it to mitigate such attacks on the main pages of their sites. However, if CSP is not installed on some pages of the same site, this can potentially leak to CSP violations due to the inconsistency with SOP when another page from the same origin is included as an iframe (see Figure 1). In our database, for each site, we recorded its home page, a number of linked pages and iframes from the same site. This allows us to analyse how CSP is adopted at every popular site by checking the presence of CSP on every crawled page and iframe of each site. To do so, we analyse the extracted CSPs: $\mathcal{C}$ for the home page, $\mathcal{C}_{L}$ for linked pages, $\mathcal{C}_{F}$ for home page iframes, and $\mathcal{C}_{L F}$ for linked pages iframes.

\subsubsection{CSP violations detection}

To detect possible CSP violations due to SOP, we have analysed home pages and linked pages from the same site, as well as iframes embedded into them.

\section{CSP Selection}

To detect CSP violations, we first remove all the sites where no parent page and no iframe page contains a CSP. For the remaining sites, we pointwise compare (1) the CSPs of the home pages $\mathcal{C}$ and CSPs of iframes present on these pages $\mathcal{C}_{F} ;(2)$ the CSPs of the linked pages $\mathcal{C}_{L}$ and CSPs of their iframes $\mathcal{C}_{L F}$. To check whether a parent page CSP and an iframe CSP are equivalent, we have applied the CSP comparison algorithm (Figure 2

CSP Preprocessing We first normalise each CSP policy, by splitting it into its directives.

- If default-src directive is present (default-src is a fallback for most of the other directives), then we extract the source list $s$ of default-src. We analyse which directives are missing in the CSP, and explicitly add them with the source list $s$.

- If default-src directive is absent, we extract missing directives from the CSP. In this case, there are no restrictions in CSP for every absent directive. We therefore explicitly add them with the most permissive source list. A missing script-src is assigned * 'unsafe-inline' 'unsafe-eval' as the most permissive source list [28].

- In each source list, we modify the special keywords: (i) 'self' is replaced with the origin of the page containing the CSP; (ii) in case of 'unsafe-inline' with hash or nonce, we remove 'unsafe-inline' from the directive since it will be ignored by the CSP2. (iii) 'none' keywords are removed from all the directives; (iv) nonces and hashes are removed from all the directives since they cannot be compared; (iv) each whitelisted domain is extended with a list of schemes and port numbers from the URL of the page includes the $\mathrm{CSP}^{4}$

\footnotetext{
${ }^{4}$ For example, according to CSP2, if the page scheme is https, and a CSP contains a source example.com, then the user agent should allow content only from https://example.com, while if the current scheme is http, it would allow both http://example.com and https://example.com.
} 


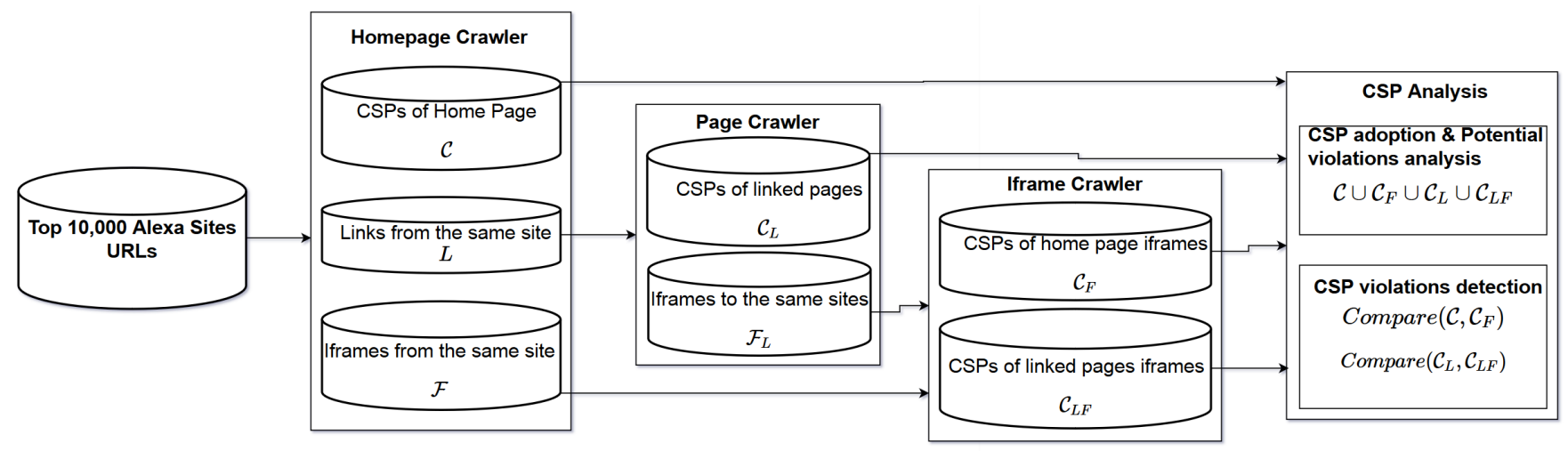

Figure 2: Data Collection and Analysis Process

\begin{tabular}{|l|l|}
\hline Sites successfully crawled & 9,885 \\
\hline Pages visited & $1,090,226$ \\
\hline Pages with iframe(s) from the same site & 648,324 \\
\hline Pages with same-origin iframe(s) & 92,430 \\
\hline $\begin{array}{l}\text { Pages with same-origin iframe(s) where } \\
\text { page and/or iframe has CSP }\end{array}$ & 692 \\
\hline Pages with CSP & $21,961(2.00 \%)$ \\
\hline Sites with CSP on home page & $228(2.3 \%)$ \\
\hline Sites with CSP on some pages & $523(5.29 \%)$ \\
\hline
\end{tabular}

Table 2: Crawling statistics

CSP Comparison We compare all the directives present in the two CSPs to identify whether the two policies require the same restrictions. Whenever the two CSPs are different, our algorithm returns the names of directives that do not match. The demonstration of the comparison is accessible on 2]. For each directive in the policies we compare the source lists and the algorithm proceeds if the elements of the lists are identical in the normalised CSPs.

\subsubsection{Limitations}

Our methodology and results have two(2) limitations that we explain here.

User interactions The automatic crawling process did not include any real-user-like interactions with top sites. As such the set of iframes and links URLs we have analysed is an underestimate of all links and iframes a site may contain.

Pairs of (parent-iframe) In this study, we consider CSP violations in same origin (parent, iframe) couples only. Their are though further combinations such as couples of sibling iframes in a parent page that we could have considered. Overall, our results are conservative, since the problem might have been worst without those limitations.

\subsection{Results on CSP Adoption}

The crawling of Alexa top 10,000 sites was performed in the end of August, 2016. To extract several pages from the same site, we have also crawled all the links and iframes on a page that point to the same site. In total, we have gathered 1,090,226 from 9,885 different sites. On median, from each site we extracted 45 pages, with a maximum number of 9,055 pages found on tuberel.com Our crawling statistics is presented in Table 2. More than half of the pages contain an iframe, and $13 \%$ of pages do contain an iframe from the same site. This indicates the potential surface for

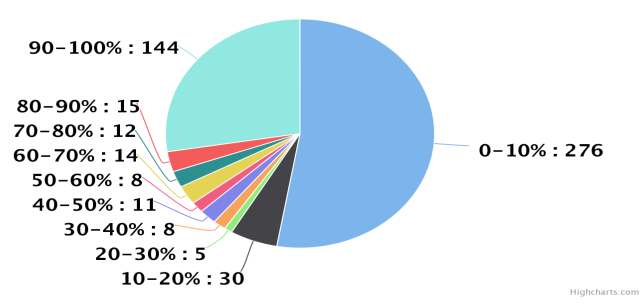

Figure 3: Percentage of pages with CSP per site

the CSP violations, when at least one page on the site has a CSP installed. We discuss such potential CSP violation in details in Section 3.3.3 Similarly to previous works on CSP adoption 22, 5, we have found that CSP is present on only 228 out of 9,885 home pages $(2.31 \%)$. While extending this analysis to almost a million pages, we have found a similar rate of CSP adoption (2.00\%).

Differently from previous studies that anlaysed only home pages, or only pages in separation, we have analysed how many sites have at least some pages that adopted CSP. We have grouped all pages by sites, and found that $5.29 \%$ of sites contain some pages with CSPs. It means that CSP is more known by the website developers, but for some reason is not widely adopted on all the pages of the site.

We have then analysed how many pages on each site have adopted CSPs. For each of 523 sites, we have counted how many pages (including home page, linked pages and iframes) have CSPs. Figure 3 shows that more than half of the sites have a very low CSP adoption on their pages: on 276 sites out of 529 , CSP is installed on only $0-10 \%$ of their pages. This becomes problematic if other pages without CSP are not XSS-free. However, it is interesting to note that around a quarter of sites do profit from CSP by installing it on 90$100 \%$ of their pages.

\subsection{Results on CSP violations due to SOP}

As described in Section 2.1.4, we distinguish several categories of CSP violations when a parent page and an iframe on this page are from the same origin according to SOP. To account for possible CSP violations, we only consider cases when either parent, or iframe, or both have a CSP installed. From all the 21,961 pages that have CSP installed, we have removed the pages, where CSPs are in report-only mode, having left 18,035 pages with CSPs in enforcement mode.

Table 3 presents possible CSP violations due to SOP. 


\begin{tabular}{|c|l|l|l|}
\cline { 2 - 4 } \multicolumn{1}{c|}{} & Same-origin parent-iframe & Possible to relax origin & Total \\
\hline Only parent page has CSP & $\mathbf{8 3}$ & $\mathbf{1 3 8 8}$ & 1471 \\
\hline Only iframe has CSP & $\mathbf{1 6}$ & $\mathbf{2 4 0}$ & 256 \\
\hline Different CSPs in parent page and iframe & $\mathbf{7 0}$ & $\mathbf{4 4}$ & 114 \\
\hline No CSP violations & 551 & 109 & 660 \\
\hline \hline CSP violations total & $\mathbf{1 6 9}(\mathbf{2 3 . 5 \% )}$ & $\mathbf{1 6 7 2}(\mathbf{9 4 \% )}$ & 1841 \\
\hline
\end{tabular}

Table 3: Statistics CSP violations due to Same-Origin Policy

\begin{tabular}{|c|l|l|}
\cline { 2 - 3 } \multicolumn{1}{c|}{} & Same-origin parent-iframe & Possible to relax origin \\
\hline Only parent page CSP & yandex.ru & twitter.com, yandex.ru, mail.ru \\
\hline Only iframe CSP & amazon.com, imdb.com & $-^{*}$ \\
\hline Different CSP & twitter.com & $-{ }^{*}$ \\
\hline
\end{tabular}

Table 4: Sample of sites with CSP violations due to Same-Origin Policy

We have extracted the parent-iframe couples that might cause a CSP violation because either (1) only parent or only iframe installed a CSP, or (2) both installed different CSPs. First, to account for direct violations because of SOP, we distinguish couples where parent and iframe are from the same origin (columns 2,3), we have found 720 cases of such couples. Second, we analyse possible CSP violations due to origin relaxation: we have collected 1781 couples that are from different origins but their origins can be relaxed by document.domain API (see more in Section 2.1.3 - these results are shown in column 3 .

In Table 4 we present the names of the domains out of top 100 Alexa sites, where we have found different CSP violations. Each company in this table have been notified about the possible CSP violation. Concrete examples of the page and iframe URLs and their corresponding CSPs for each such violation can be found in the corresponding technical report 18 . All the collected data is available online 2 .

CSP violations in presence of document.domain According to our results, in presence of document.domain, $94 \%$ of (parent, iframe) pages can have their CSP violated. Those violations can occur only if both parent and iframes pages execute document.domain to the same top level domain. Thus, our result is an over-approximation, assuming that document.domain is used in all of those pages and iframes. According to 1], document.domain is used in less than $3 \%$ of web pages.

\subsubsection{Only parent page or only iframe has CSP}

We first consider a scenario when a parent page and an iframe are from the same origin, but only one of them contains a CSP. Intuitively, if only a parent page has CSP, then an iframe can violate CSP by executing any code and accessing the parent page's DOM, inserting content, access cookies etc. Among 720 parent-iframe couples from the same origin, we have found 83 cases $(11.5 \%)$ when only parent has a CSP, and 16 cases $(2.2 \%)$ when only iframe has a CSP. These CSP violations originate from 13 (for parent) and 4 (for iframe) sites. For example, such possible violations are found on some pages of amazon.com, yandex.ru and imdb.com (see Table 4). CSP of a parent or iframe may also be violated because of origin relaxation. We have identified 1388 cases (78\%) of parent-iframe couples where such violation may occur because CSP is present only in the parent page. This was observed on 20 different sites, including twitter.com, yan-

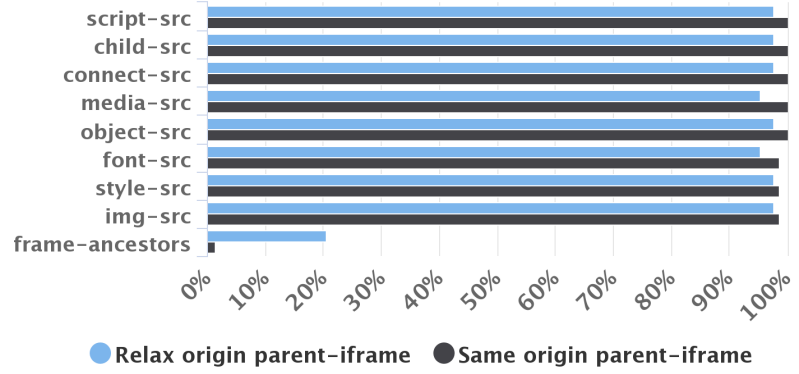

Figure 4: Differences in CSP directives for parent and iframe pages

dex.ru and others. Finally, in 240 cases (13.5\%) only iframe has CSP installed, which was found on 11 different sites. We manually checked the parent and iframes involved in CSP violations for sites in Table 4 . In all of those sites, either the parent or the iframe page is a login page 2]. We furthermore checked how effective are the CSP of those pages, using CSPEvaluator 5 proposed by Lukas et al. 22. and found out that the CSP policies involved in these are moreover all bypassable.

\subsubsection{Parent and iframe have different CSPS}

In a case when a page and iframe are from the same origin, but their corresponding CSPs are different, may also cause a violation of CSP. From the 720 same-origin parent-iframe couples, we have found 70 cases (9.7\%) (from 3 sites) when their CSPs differ, and for an origin relaxation (from 6 sites) case, we have identified only 44 such cases $(2.5 \%)$. This setting was found on some pages of twitter.com for instance.

We have further analysed the differences in CSPs found on parent and iframe pages. For all the 114 pairs of parentiframe (either same-origin or possible origin relaxation), we have compared CSPs they installed, directive-by-directive. Figure 4 shows that every parent CSP and iframe CSP differ on almost every directive - between $90 \%$ and $100 \%$. The only exception is frame-ancestors directive, which is almost the same in different parent pages and iframes. If properly set, this directive gives a strong protection against clickjacking attacks, therefore all the pages of the same origin are equally protected.

\footnotetext{
${ }^{5}$ https://csp-evaluator.withgoogle.com/
} 


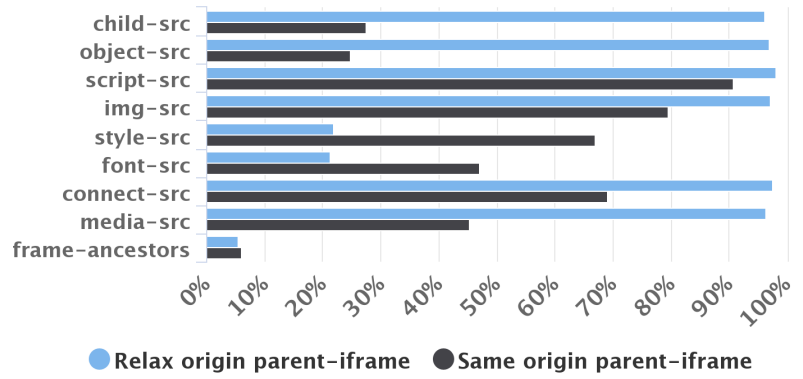

Figure 5: Differences in CSP directives for sameorigin and relaxed origin pages

\subsubsection{Potential CSP violations}

A potential CSP violation may happen when in a site, either some pages have CSP and some others do not, or pages have different CSP. When those pages get nested as parentiframe, we can run into CSP violations, just like in the direct CSP violations cases we have just reported above. To analyse how often such violations may occur, we have analysed the 18,035 pages that have CSP in enforcement mode. These pages originate from 729 different origins spread over 442 sites. Table 5 shows that $72 \%$ of CSPs (12,899 pages) can be potentially violated, and these CSPs originate from pages of 379 different sites $(85.75 \%)$. To detect these violations, for each page with a CSP in our database, we have analysed whether there exists another page from the same origin, that does not have CSP. This page could embed the page with CSP and violate it because of SOP. We have detected 4381 such pages (24\%) from 197 origins. Similarly, we detected 1223 pages $(7 \%)$ when there are same-origin pages with a different CSP. Similarly, we have analysed when potential CSP violations may happen due to origin relaxation. We have detected 4728 pages (26\%), whose CSP may be violated because of other pages with no CSP, and 2567 pages (14\%), whose CSP may be violated because of different CSP on other relaxed-origin pages.

For the pages that have different CSPs, we have compared how much CSPs differ. Figure 5 shows that CSPs mostly differ in script-src directive, which protects pages from XSS attacks. This means, that if one page in the origin does whitelist an attacker's domain or an insecure endpoints 22], all the other pages in the same origin become vulnerable because they may be inserted as an iframe to the vulnerable page and their CSPs can be easily violated.

\subsection{Responses of websites owners}

We have reported those issues to a sample of sites owners, using either HackerOne $6^{6}$ or contact forms when available. Here are some selected quotes from our discussions with them.

"Yes, of course we understand the risk that un-
der some circumstances XSS on one domain can
be used to bypass CSP on another domain, but
it's simply impossible to implement CSP across
all (few hundreds) domains at once on the same
level. We are implementing strongest CSP cur-
rently possible for different pages on different do-
mains and keep going with this process to protect
all pages, after that we will strengthen the CSP.

${ }^{6}$ https://hackerone.com
We believe it's better to have stronger CSP policy where possible rather than have same weak CSP on all pages or not having CSP at all. Having in mind there are hundreds of domains within mail.ru, at least few years are required before all pages on all domains can have strong CSP." Mail.ru

"[...] the sandbox is a defense in depth mitigation[...]. We definitely don't allow relaxing document.domain on www.dropbox.com[...]" - Dropbox.com

"While this is an interesting area of research, are you able to demonstrate that this behavior is currently exploitable on Twitter? It appears that the behavior you have described can increase the severity of other vulnerabilities but does not pose a security risk by itself. Is our understanding correct? [...]We consider this to be more of a defensive in depth and will take into account with our continual effort to improve our CSP policy" - Twitter.com

"I believe we understand the risk as you've described it." - Imdb.com

\section{AVOIDING CSP VIOLATIONS}

Preventing CSP violations due to SOP can be achieved by having the same effective CSP for all same-origin pages in a site, and prevent origin relaxation.

Origin-wide CSP: Using CSP for all same-origin pages can be manually done but this solution is error-prone. A more effective solution is the use of a specification such as Origin Policy 27] in order to set a header for the whole origin.

Preventing Origin Relaxation: Having an origin-wide CSP is not enough to prevent CSP violations. By using origin relaxation, pages from different origins can bypass the SOP 17. Many authors provide guidelines on how to design an effective CSP 22]. Nonetheless, even with an effective CSP, an embedded page from a different origin in the same site can use document.domain to relax its origin. Preventing origin relaxation is trickier.

Programmatically, one could prevent other scripts from modifying document. domain by making a script run first in a page 20. The first script that runs on the page would be:

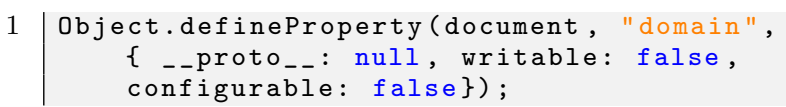

A parent page can also indirectly disable origin relaxation in iframes by sandboxing them. This can be achieved by using sandbox as an attribute for iframes or as directive for the parent page CSP. Unfortunately, an iframe cannot indirectly disable origin relaxation in the page that embeds it. However, the frame-ancestors directive of CSP gives an iframe control over the hosts that can embed it. Finally, a more robust solution is the use of a policy to deprecate document.domain as proposed in the draft of Feature policy 29]. The feature policy defines a mechanism that allows developers to selectively enable and disable the use of various browser features and APIs.

Iframe sandboxing: Combining attribute allow-scripts and allow-same-origin as values for sandbox successfully 


\begin{tabular}{|l|l|l|l|}
\cline { 2 - 4 } \multicolumn{1}{c|}{} & Pages & Origins & Sites \\
\hline A same origin page has no CSP & 4381 & 197 & 197 \\
\hline A same origin page has a different CSP & 1223 & 23 & 23 \\
\hline Total Potential violations due to same origin pages & $\mathbf{5 6 0 4} \mathbf{( 3 1 . 1 \% )}$ & - & - \\
\hline \hline A same origin (after relaxation) page has no CSP & 4728 & 340 & 183 \\
\hline A same origin (after relaxation) has a different CSP & 2567 & 135 & 44 \\
\hline Total Potential violations due to same origin (after relaxation & $\mathbf{7 2 9 5 ( 4 0 . 4 \% )}$ & - & - \\
\hline \hline Potential violations total & $12899(72 \%)$ & $591(81 \%)$ & $379(52 \%)$ \\
\hline
\end{tabular}

Table 5: Potential CSP violations in pages with CSP

disables document.domain in an iframe 7 We recommend the use of sandbox as a CSP directive, instead of an HTML iframe attribute. The first reason is that sandbox as a CSP directive, automatically applies to all iframes that are in a page, avoiding the need to manually modify all HTML iframe tags. Second, the sandbox directive is not programmatically accessible to potentially malicious scripts in the page, as is the case for the sandbox attribute (which can be removed from an iframe programmatically, replacing the sandboxed iframe with another identical iframe but without the sandbox attribute).

Limitations An origin-wide CSP (the same CSP for all same origin pages) can become very liberal if all same origin pages do not require the same restrictions. In order to implement the solution we propose, one needs to consider the intended relation between a parent page and an iframe page, in presence of CSP. In the case where the two(2) pages should be allowed direct access to each other content, then, since same origin pages can bypass page-specific security characteristics [9], the solution is to have the same CSP for both the page and the iframe. However, if direct access to each other content is not a required feature, one can keep different CSPs in parent and iframe, or have no CSP at all in one of the parties, but their contents should be isolated from each other. The solution here is to use sandboxing. Nonetheless, there are other means (such as postMessage) by which one can securely achieve communication between the pages.

\section{INCONSISTENT IMPLEMENTATIONS}

Combining origin-wide CSP with allow-scripts sandbox directive would have been sufficient at preventing the inconsistencies between CSP and the same origin policy. Unfortunately, we have discovered that for some browsers, this solution is not sufficient. Starting from HTML5, major browsers, apart from Internet Explorer, supports the new srcdoc attribute for iframes. Instead of providing a URL which content will be loaded in an iframe, one provides directly the HTML content of the iframe in the srcdoc attribute. According to CSP2 28], §5.2, the CSP of a page should apply to an iframe which content is supplied in a srcdoc attribute. This is actually the case for all majors browsers, which support the srcdoc attribute. However, there is a problem when the sandbox attribute is set to an srcdoc iframe.

\footnotetext{
${ }^{7}$ We found out that dropbox.com actually puts sandbox attribute for all its iframes, and therefore avoids the possible CSP violations. We have had a very interesting discussion on Hackerone.com with Devdatta Akhawe, a Security Engineer at Dropbox, who told us more about their security practices regarding CSP in particular.
}

Webkit-based ${ }^{8}$ and Blink-based ${ }^{9}$ browsers (Chrome, Chromium, Opera) always comply with CSP. The CSP of a page will apply to all srcdoc iframes, even in those iframes which have a different origin than that of the page, because they are sandboxed without allow-same-origin .

In contrast, we noticed that in Gecko-based browsers (Mozilla Firefox), the CSP of the page applies to that of the srcdoc iframe if and only if allow-same-origin is present as value for the attribute. Otherwise it does not apply. The problem with this choice is the following. A third party script, whitelisted by the CSP of the page, can create a srcdoc iframe, sandboxing it with allow-scripts only, and load any resource that would normally be blocked by the CSP of the page if applied in this iframe. This way, the third party script successfully bypasses the restrictions of the CSP of the page. Even though loading additional scripts is considered harmless in the upcoming version 3 [26, 22 of CSP, this specification says nothing about violations that could occur due to the loading of other resources inside a srcdoc sandboxed iframe, like resources whitelisted by object-src directive for instance, additional iframes etc.

We have notified the W3C, and the Mozilla Security Group. Daniel Veditz, a lead at Mozilla Security Group, recognizes this as a bug and explains:
"Our internal model only inherits CSP into same- origin frames (because in theory you're otherwise leaking info across origin boundaries) and iframe sandbox creates a unique origin. Obviously we need to make an exception here (I think we man- age to do the same thing for src=data: sandboxed frames)."

CSP specification and srcdoc iframes The problem of imposing a CSP to an unknown page is illustrated by the following example 25. If a trusted third party library, whitelisted by the CSP of the page, uses security libraries inside an isolated context (by sandboxing them in a srcdoc iframe, setting allow-scripts as sole value for the sandbox) then, the page's CSP will block the security libraries and possibly introduce new vulnerabilities. Because of this, it was unclear to us the intent of CSP designers regarding srcdoc iframes. Mike West, one of the CSP editors at the W3C and also Developer Advocate in Google Chrome's team, clarified this to us:

"I think your objection rests on the notion of the same-origin policy preventing the top-level document from reaching into it's sandboxed child. That seems accurate, but it neglects the bigger

\footnotetext{
${ }^{8}$ https://en.wikipedia.org/wiki/WebKit

${ }^{9}$ https://en.wikipedia.org/wiki/Blink_(web_engine)
} 
picture: srcdoc documents are produced entirely from the top-level document context. Since those kinds of documents are not delivered over the network, they don't have the opportunity to deliver headers which might configure their settings. We impose the parent's policy in these cases, because for all intents and purposes, the srcdoc document is the parent document."

\section{RELATED WORK}

CSP has been proposed by Stamm et al. [19] as a refinement of SOP 3], in order to help mitigate Cross-Site-Scripting 30 and data exfiltration attacks. The second version 28 of the specification is supported by all major browsers, and the third version 26] is under active development. Even though CSP is well supported [5], its endorsement by web sites is rather slow. Weissbacher et al. 24] performed the first large scale study of CSP deployment in top Alexa sites, and found that around $1 \%$ of sites were using CSP at the time. A more recent study by Calzavara et al. [5], show that nearly $8 \%$ of Alexa top sites now have CSP deployed in their front pages. Another recent study, by Weichselbaum et al. 22 come with similar results to the study of Weissbacher et al. 24. Our work extends previous results by analysing the adoption of CSP by site not only considering front pages but all the pages in a site. Almost all authors agree that CSP adoption is not a straightforward task, and lots of (manual) effort are needed in order to reorganize and modify web pages to support CSP.

Therefore, in order to help web sites developers in adopting CSP, Javed proposed CSP Aider, 10 that automatically crawl a set of pages from a site and propose a site-wide CSP. Patil and Frederik 14 proposed UserCSP, a framework that monitors the browser internal events in order to automatically infer a CSP for a web page based on the loaded resources. Pan et al. 13 propose CSPAutoGen, to enforce CSP in real-time on web pages, by rewriting them on the fly client-side. Weissbacher et al. 24 have evaluated the feasibility of using CSP in report-only mode in order to generate a CSP based on reported violations, or semi-automatically inferring a CSP policy based on the resources that are loaded in web pages. They concluded that automatically generating a CSP is ineffective. A difficulty which remains is the use of inline scripts in many pages. The first solution is to externalize inline scripts, as can be done by systems like deDacota 6. Kerschbaumer et al. 12 find that too many pages are still using 'unsafe-inline' in their CSPs. They propose a system to automatically identify legitimate inline scripts in a page, thereby whitelisting them in the CSP of the underlying page, using script hashes.

Another direction of research on CSP, has been evaluating its effectiveness at successfully preventing content injection attacks. Calzavara et al. 5. found out that many CSP policies in real web sites have errors including typos, illformed or harsh policies. Even when the policies are well formed, they have found that almost all currently deployed CSP policies are bypassable because of a misunderstanding of the CSP language itself. Patil and Frederik found similar errors in their study 14. Hausknecht et al. 7. found that some browser extensions, modified the CSP policy headers, in order to whitelist more resources and origins. Van Acker et al. 4] have shown that CSP fails at preventing data exfiltration specially when resources are prefetched, or in pres- ence of a CSP policy in the HTML meta tag, because the order in which resources are loaded in a web application is hard to predict. Johns 11] proposed hashes for static scripts, and PreparedJS, an extension for CSP, in order to securely handle server-side dynamically generated scripts based on user input. Weichselbaum et al. 22 have extended nonces and hashes, introduced in CSP level 2 28, to remote scripts URLs, specially to tackle the high prevalence of insecure hosts in current CSP policies. Furthermore, they have introduced strict-dynamic. This new keyword states that any additional script loaded by a whitelisted remote script URL is considered a trusted script as well. They also provide guidelines on how to build an effective CSP. Jackson and Barth 9 have shown that same origin pages can bypass page-specific policies, like CSP. Though, their work predates CSP. To the best of our knowledge, we are the first to explore the interactions between CSP and SOP and report possible CSP violations.

\section{CONCLUSIONS}

In this work, we have revealed a new problem that can lead to violations of CSP. We have performed an in-depth analysis of the inconsistency that arises due to CSP and SOP and identified three cases when CSP may be violated.

To evaluate how often such violations happen, we performed a large-scale analysis of more than 1 million pages from 10,000 Alexa top sites. We have found that $5.29 \%$ of sites contain pages with CSPs (as opposed to $2 \%$ of home pages in previous studies).

We have also found out that $72 \%$ of current web pages with CSP, are potentially vulnerable to CSP violations. This concerns 379 (72.46\%) sites that deploy CSP. Further analysing the contexts in which those web pages are used, our results show that when a parent page includes an iframe from the same origin according to SOP, in $23.5 \%$ of cases their CSPs may be violated. And in the cases where document.domain is required in both parent and iframes, we identified that such violations may occur in $94 \%$ of the cases.

We discussed measures to avoid CSP violations in web applications by installing an origin-wide CSP and using sandboxed iframes. Finally, our study reveals an inconsistency in browsers implementation of CSP for srcdoc iframes, that appeared to be a bug in Mozilla Firefox browsers.

\section{Acknowledgments}

The authors would like to thank the WebAppSec W3C Working Group for useful pointers to related resources at the early stage of this work, Mike West for very insightful discussions that considerably helped improve this work, Devdatta Akhawe for discussing some security practices at Dropbox, and anonymous reviewers and Stefano Calzavara for their valuable comments and suggestions. 


\section{REFERENCES}

[1] Chrome Platform Status. https://www.chromestatus.com/metrics/feature/ popularity\#DocumentSetDomain

[2] CSP violations online. https://webstats.inria.fr?cspviolations

[3] Same Origin Policy. https: //www.w3.org/Security/wiki/Same_Origin_Policy.

[4] S. V. Acker, D. Hausknecht, and A. Sabelfeld. Data Exfiltration in the Face of CSP. In X. Chen, X. Wang, and X. Huang, editors, Proceedings of the 11th ACM on Asia Conference on Computer and Communications Security, AsiaCCS 2016, Xi'an, China, May 30 - June 3, 2016, pages 853-864. ACM, 2016.

[5] S. Calzavara, A. Rabitti, and M. Bugliesi. Content Security Problems?: Evaluating the Effectiveness of Content Security Policy in the Wild. In Weippl et al. 23], pages 1365-1375.

[6] A. Doupé, W. Cui, M. H. Jakubowski, M. Peinado, C. Kruegel, and G. Vigna. deDacota: toward preventing server-side XSS via automatic code and data separation. In A. Sadeghi, V. D. Gligor, and M. Yung, editors, 2013 ACM SIGSAC Conference on Computer and Communications Security, CCS'13, Berlin, Germany, November 4-8, 2013, pages 1205-1216. ACM, 2013.

[7] D. Hausknecht, J. Magazinius, and A. Sabelfeld. May I? - Content Security Policy Endorsement for Browser Extensions. In M. Almgren, V. Gulisano, and F. Maggi, editors, Detection of Intrusions and Malware, and Vulnerability Assessment - 12th International Conference, DIMVA 2015, Milan, Italy, July 9-10, 2015, Proceedings, volume 9148 of Lecture Notes in Computer Science, pages 261-281. Springer, 2015.

[8] A. Hidayat. PhantomJS Headless Browser, 2010-2016.

[9] C. Jackson and A. Barth. Beware of Finer-Grained Origins. In Web 2.0 Security and Privacy (W2SP 2008), 2008.

[10] A. Javed. CSP Aider: An Automated Recommendation of Content Security Policy for Web Applications. In IEEE Oakland Web 2.0 Security and Privacy (W2SP'12), 2012.

[11] M. Johns. PreparedJS: Secure Script-Templates for JavaScript. In Detection of Intrusions and Malware, and Vulnerability Assessment - 10th International Conference, DIMVA 2013, Berlin, Germany, July 18-19, 2013. Proceedings, pages 102-121, 2013.

[12] C. Kerschbaumer, S. Stamm, and S. Brunthaler. Injecting CSP for Fun and Security. In O. Camp, S. Furnell, and P. Mori, editors, Proceedings of the 2nd International Conference on Information Systems Security and Privacy (ICISSP 2016), Rome, Italy, February 19-21, 2016., pages 15-25. SciTePress, 2016.

[13] X. Pan, Y. Cao, S. Liu, Y. Zhou, Y. Chen, and T. Zhou. CSPAutoGen: Black-box Enforcement of Content Security Policy upon Real-world Websites. In Weippl et al. 23], pages 653-665.

[14] K. Patil and B. Frederik. A Measurement Study of the Content Security Policy on Real-World Applications. I. J. Network Security, 18(2):383-392, 2016.
[15] N. Perriault. CasperJS navigation and scripting tool for PhantomJS, 2011-2016.

[16] G. Rydstedt, E. Bursztein, D. Boneh, and C. Jackson. Busting frame busting: a study of clickjacking vulnerabilities at popular sites. In in IEEE Oakland Web 2.0 Security and Privacy (W2SP 2010), 2010.

[17] K. Singh, A. Moshchuk, H. J. Wang, and W. Lee. On the Incoherencies in Web Browser Access Control Policies. In 31st IEEE Symposium on Security and Privacy, SEPP 2010, 16-19 May 2010,

Berleley/Oakland, California, USA, pages 463-478, 2010.

[18] D. F. Some, N. Bielova, and T. Rezk. On the Content Security Policy violations due to the Same-Origin Policy. Technical report. http://www-sop.inria.fr/ members/Nataliia.Bielova/papers/CSP-SOP.pdf

[19] S. Stamm, B. Sterne, and G. Markham. Reining in the web with content security policy. In M. Rappa, P. Jones, J. Freire, and S. Chakrabarti, editors, Proceedings of the 19th International Conference on World Wide Web, WWW 2010, Raleigh, North Carolina, USA, April 26-30, 2010, pages 921-930. ACM, 2010.

[20] N. Swamy, C. Fournet, A. Rastogi, K. Bhargavan, J. Chen, P. Strub, and G. M. Bierman. Gradual typing embedded securely in JavaScript. In S. Jagannathan and P. Sewell, editors, The 41st Annual ACM SIGPLAN-SIGACT Symposium on Principles of Programming Languages, POPL '14, San Diego, CA, USA, January 20-21, 2014, pages 425-438. ACM, 2014.

[21] A. van Kesteren. Cross Origin Resource Sharing. W3C Recommendation, 2014.

[22] L. Weichselbaum, M. Spagnuolo, S. Lekies, and A. Janc. CSP Is Dead, Long Live CSP! On the Insecurity of Whitelists and the Future of Content Security Policy. In Weippl et al. [23], pages 1376-1387.

[23] E. R. Weippl, S. Katzenbeisser, C. Kruegel, A. C. Myers, and S. Halevi, editors. Proceedings of the 2016 ACM SIGSAC Conference on Computer and Communications Security, Vienna, Austria, October 24-28, 2016. ACM, 2016.

[24] M. Weissbacher, T. Lauinger, and W. K. Robertson. Why Is CSP Failing? Trends and Challenges in CSP Adoption. In Research in Attacks, Intrusions and Defenses - 17th International Symposium, RAID 2014, Gothenburg, Sweden, September 17-19, 2014. Proceedings, pages 212-233, 2014.

[25] M. West. Content Security Policy: Embedded Enforcement, 2016.

[26] M. West. Content Security Policy Level 3. W3C Working Draft, 2016.

[27] M. West. Origin Policy. A Collection of Interesting Ideas, 2016.

[28] M. West, A. Barth, and D. Veditz. Content Security Policy Level 2. W3C Candidate Recommendation, 2015.

[29] M. West and I. Grigorik. Feature Policy. W3C Draft Community Group Report, 2016.

[30] I. Yusof and A. K. Pathan. Mitigating Cross-Site Scripting Attacks with a Content Security Policy. IEEE Computer, 49(3):56-63, 2016. 